\title{
An Overview of the Actin-Myosin Interaction
}

\author{
David D. Thomas and Osha Roopnarine ${ }^{1}$
}

It is proposed that force generation in muscle contraction occurs through a transition from a weakly bound state to a strongly bound state of myosin on actin during the ATPase reaction cycle. The chapters in this book probe the structural details of these interactions, with particular attention to the specific sites of interaction on each protein, the structural and dynamics properties of the bound complexes, and the differences between weak and strong interactions.

A structural model of the actin-S1 complex, based on crystal structures of the actin monomer and S1, has provided clues about the weak-binding (primarily ionic) and strong-binding (primarily hydrophobic) interactions between these two proteins (Rayment et al., 1993, Schroder et al., 1993; Milligan, 1996; Vale 1996; Mendelson and Morris, 1997). Several specific sites on myosin have been implicated in these interactions with actin: (1) the residues 626-647 of skeletal myosin (referred to as either loop 2 or as the actinbinding loop) are proposed to interact with the $\mathrm{N}$-terminal residues in actin (residues 1-4) during the weak-to-strong transition of muscle contraction; (2) the helix-loop-helix, residues Gly516-His558 of the lower 50-kDa domain of myosin, is proposed to interact with actin through both ionic and hydrophobic interactions; (3) the residues Arg405-Lys415 of myosin form a flexible loop that appears to bind to actin with high affinity and are important for the actinactivated ATPase activity of myosin; and (4) the residues 567-578 (called loop 3 ) of myosin are proposed to be involved in hydrophobic interactions with residues $99 / 100$ of actin.

The seven chapters in this section review recent studies on the actin-myosin interaction. To elucidate the interactions that occur in the actin-myosin interface, we must perturb the functional sites in this region of both proteins. This can be done by (1) using probes on either actin or myosin to directly measure dynamic interactions during the ATPase cycle (see Chapters by Thomas and colleagues, Yengo and Berger); (2) creating specific mutations in either actin or myosin to determine its functional effect on the actomyosin ATPase, binding, and actin motility (the chapters by Doyle and Reisler; Asugawa and Sutoh; Roopnarine); (3) capturing the actin-myosin complex by chemically

\footnotetext{
${ }^{1}$ Department of Biochemistry, University of Minnesota Medical School, Minneapolis, Minnesota 55455 , USA
} 
cross-linking the complex in different chemical states using nucleotide analogs (chapter by Chaussepied and Van Dijk); and (4) simultaneously probing the mechanical and conformational changes that occur during the actomyosin interaction (see Chapter by Ishii and colleagues).

\section{Structural Changes in Actin and Myosin Due to Their Strong and Weak Interactions}

Conventional models suggest that the structural state of myosin is determined by the state of its bound nucleotide, which in turn determines whether it binds weakly or strongly to actin. In this first chapter, Thomas et al. ask the following questions: Do the structure and dynamics of actin depend on the binding of the myosin head and/or on the structural state (weak or strong) of the bound myosin head? Do the structure and dynamics of myosin depend on interaction with actin? This balanced approach to actin-myosin dynamics is made possible by spectroscopic analysis of site-specific spectroscopic probes attached to either actin or myosin. The results of these studies indicate that weak binding of myosin to actin produces a dynamically disordered complex that does not greatly perturb the structural dynamics of either actin or myosin, while the formation of the strong complex greatly restricts this disorder. It is likely that this disorder-to-order transition provides the driving force for actomyosin energy transduction.

\section{Fluorescence Resonance Energy Transfer in Actomyosin Complexes}

Yengo and Berger measured the distance between Cys374 of actin and Lys553 of $\mathrm{S} 1$ as a function of actin concentration using fluorescence probes. They found an increase of $5.6 \AA$ when the S1/actin ratio increased above 0.5 under rigor conditions. These results suggest that a conformational change occurs in the $\mathrm{C}$-terminus of actin due to the interaction of adjacent actin monomers that are bound to myosin S1. These results support the proposal that the myosin head interacts with two actin monomers (this concept is further discussed in the Chapter by Chaussepied and Van Dijk).

\section{Insights into Actomyosin Interactions from Actin Mutations}

Doyle and Reisler discuss the properties of actin mutants expressed and purified from either yeast or slime mold. This is a comprehensive review on the key residues of actin that have been implicated in either the weak- or strongbinding interaction with myosin. They review the biochemical studies of the mutated acidic actin residues (D24/D25 and E99/E100) that have been pro- 
posed to be involved in weakly bound states, which showed that the weakbinding states must be significantly populated in order to produce strongly bound states that generate force. They also summarize the results that clearly demonstrate the importance of the acidic N-terminal residues (2-5) in activating the myosin S1 ATPase. The actin residues that are involved in the hydrophobic interactions with myosin are also reviewed. Finally, the importance of the C-terminus of actin, the residues 262-274, and the segment (360-364) that is proposed to interact with the N-terminus of the alkali (or essential) light chain of myosin is discussed in this chapter.

They focused on three pairs of acidic residues on subdomain 1 of yeast actin (Asp2/Glu4, Asp24/Asp25, Glu99/Glu100) to show that they play an important role in the weak binding interactions with myosin. Using site-directed mutagenesis, they increased the charge of the $\mathrm{N}$-terminus of yeast actin to be equivalent with that of rabbit skeletal actin, which resulted in increased $V_{\max }$ of the mutant actin. Their results with the N-terminal actin mutants suggest that the $\mathrm{N}$-terminus may play a role in the transition of the weak to strongly bound states of myosin. The actin "charge" mutants Asp-Asn-Glu-Gln (DNEQ), $\triangle \mathrm{DSE}$, Asp24Ala/Asp25Ala, and Glu99Ala/Glu100Ala had lower $V_{\max }$ for actinactivated ATPase and actin motility but had the same rigor binding to $S 1$ as the wild type actin. These results suggest that the actin mutants decreased the population of weakly bound complexes, so that fewer myosin crossbridges attained the isomerization step with mutant actins (see Chapter by Doyle and Reisler, step 5 in Fig. 1).

\section{Role of Charges in the Actomyosin Complex}

Chaussepied and Van Dijk review the role of loop 2 (626-647) and 3 (567-578) of myosin during the actin-myosin interaction. They used cross-linking agents to trap the actin-myosin complex in different nucleotide states and then used chemical and proteolytic degradation to characterize the complexes. Their key result suggests that after cleavage of the $\beta-\gamma$ phosphate bond of the bound ATP (while still in the weak-binding state), loops 2 and 3 of myosin S1 interact simultaneously and with equal affinity with two different actin monomers. This was observed regardless of the bound ADP.Pi analog (AMPPNP, ADP.BEF $A D P . A l F_{4}$, or $A D P . V_{4}$ ), which is surprising because AMPPNP and ADP.BEF have been shown to induce different lever arm orientations compared to $\mathrm{ADP} . \mathrm{AlF}_{4}$, and $\mathrm{ADP}_{\mathrm{V}} \mathrm{VO}_{4}$ in the crystal structures of S1. While they were unable to clearly define the sites of interaction between actin and myosin prior to ATP hydrolysis (using ATP or ATP $\gamma$ S), they showed that in an ATP-bound state myosin S1 interacts differently from both the weakly- (ADP.Pi) and stronglybound states (rigor or ADP-bound). In the latter state, they observed an increase of myosin molecules interacting with a single actin monomer and a decrease of the electrostatic interactions that simultaneously involve loops 2 and 3. The significance of these results is that it suggests that the structural 
environment of the $\gamma \mathrm{Pi}$ in the active site alters the accessibility of the myosin loop structures for interaction with actin.

\section{The Alanine-Scanning Mutagenesis of Dictyostelium Myosin II at the Ionic Interface with Actin}

In their chapter, Asukagawa and Sutoh discuss the role of the $50-$ to $20-\mathrm{kDa}$ junction by creating three myosin mutations that may affect the actin-myosin interaction. They replaced three residues with alanine (Arg620Ala, Lys622Ala, Lys623Ala) in the 50 - to $20-\mathrm{kDa}$ junction of myosin (loop 2 or actin-binding loop). Both the physiological myosin ATPases and $\mathrm{V}_{\max }$ of the actin-activated ATPases were unaffected, but the actin-activated ATPases revealed that the $K_{m}$ for actin binding were higher than wild type myosin. The sliding velocities of actin filaments by each mutant myosin was lower than that of wild type myosin, but increased to wild type values at higher concentrations of the mutant myosin. These results suggest that each mutation in myosin induced weaker interactions of actin-myosin in the presence of ATP. Significantly, this work suggests that the charged residues of loop 2 play an important role in actin-myosin interactions such as ATP hydrolysis, actin-myosin sliding and force generation.

\section{Familial Hypertrophic Cardiomyopathic Mutations That Affect the Actin-Myosin Interaction}

Familial hypertrophic cardiomyopathy is caused by single missense mutations in seven cardiac sarcomeric proteins, with the most mutations $(>40)$ occurring in the myosin gene. The myosin mutations occur near the functional domains in the myosin head (ATP-binding site, actin-binding region, and LC-binding domain). Roopnarine reviews the functional results of several myosin mutations in its role of perturbing actin function and then correlates these results with the phenotypic manifestations of the disease in affected patients.

\section{Coupling Between Chemical and Mechanical Events and Conformation of Single Protein Molecules}

In their chapter, Ishii, Ishijima, and Yanagida address the question: Are the chemical states of ATPase hydrolysis directly and tightly coupled to the mechanical events that occur during muscle contraction or force displacement? They used a laser trap to measure the force exerted by myosin on an actin filament, and the single molecule imaging technique with fluorescently labeled ATP to simultaneously directly visualize the hydrolysis of ATP by myosin during the force displacement. This elegant combination of techniques allowed 
them to determine that the mechanical events are not always tightly coupled to chemical events, and that the energy from the ATPase reaction is stored in the actomyosin complex for later use. They also showed that the transition between metastable states is slow, which may explain the energy storage within actomyosin.

While the reconstruction of the actin-myosin S1 rigor complex has given us many clues about the actin-myosin interaction, the elucidation of the events that occur at this interface will have to be solved by biophysical and biochemical studies of the two proteins, both in solution and in the muscle fiber. This is especially important in determining the role of the highly flexible regions, such as loop 2, that are invisible to X-ray crystallography. Site-directed mutagenesis of both proteins plays a significant role in assessing the function of key residues in this region and will also allow targeted probing using various reporter groups. Spectroscopic measurements will continue to be necessary for determining dynamic events and to map the distances between residues on actin and myosin during the ATPase cycle.

\section{References}

Mendelson R, Morris EP (1997) The structure of the acto-myosin subfragment 1 complex: results of searches using data from electron microscopy and X-ray crystallography. Proc Natl Acad Sci USA 94:8533-8538

Milligan RA (1996) Protein-protein interactions in the rigor actomyosin complex. Proc Natl Acad Sci USA 93:21-22

Rayment I, Holden HM, Whittaker M, Yohn CB, Lorenz M, Holmes KC, Milligan RA (1993) Structure of the actin-myosin complex and its implications for muscle contraction. Science 261:58-65

Schroder RR, Manstein DJ, Jahn W, Holden H, Rayment I, Holmes KC, Spudich JA (1993) Threedimensional atomic model of F-actin decorated with Dictyostelium myosin S1. Nature 364:171-174

Vale RH (1996) Switches, latches, and amplifiers: common themes of G proteins and molecular markers. J Cell Biol 135:291-302 